\title{
Convergence Management System of Mobile Data Services
}

\author{
Ling Jiang ${ }^{1,2}$, Jixin Wang ${ }^{1}$ \\ 1 Department of Information Technology, Huazhong Normal University, \\ Wuhan, China 430079 \\ 2 Engineering Research Center of Education Information Technology, \\ Huazhong Normal University, Wuhan, China 430079 \\ ljiangcn@hotmail.com wjxin@mail.ccnu.edu.cn
}

\begin{abstract}
To meet the mobile communication development, the necessary choice for mobile operators is to build up a convergence management system for the value-added services, which should support multiple valueadded services openly. This paper introduced a new convergent management system for different mobile data services, which was developed by the author. And it focused on the development of convergent management system of mobile data services. It discussed the architecture of convergent management system and described three main management methods of typical data services.
\end{abstract}

\section{Introduction}

In recent years, the user number and market capacity of mobile value-added service grew rapidly, and it became the focus of all circles and many companies tried to enter to this field. In 2004, the mobile data value-added service market capacity of China was 30.8 billion yuan (RMB) with 5.3 billion (RMB) for SP market and the user number has accessed 235 million. Furthermore, after several years of development, there has been a distinctive advance in the operation idea and management mode for operators. The business mode and operation management of value-added service is also growing into maturity [1]. Nowadays the new generation mobile communication technologies such as $2.5 \mathrm{G}$ (CDMA 1X, GPRS and EDGE), 3G (UMTS, W-CDMA), bring new challenges to mobile operators and SP/CPs (Service Provider/Content Provider)[2], and also bring new profitability and complex cooperation relationships. Mobile operators usually face many new challenges when introducing a new technology or entering into an uncertain service market. It, therefore, will bring great influence to service development to establish a convergence value-added service management system. At present, mobile operators also strengthen research on mobile data value-added service management platform, and they need a reliable and suitable solution.

Please use the following format when citing this chapter:

Jiang, L., Wang, J, 2007, in IFIP International Federation for Information Processing, Volume 25l, Integration and Innovation Orient to E-Society Volume 1, Wang, W. (Eds), (Boston: Springer), pp. 330-336. 


\section{Analysis of mobile data services}

The development of mobile value-added service mainly depends on the carrying capacity of data channel in mobile communication networks. With main $2.5 \mathrm{G}$ mobile communication technologies going into commercial application, the data carrying capacity of mobile communication networks has distinct improvement. At the same time, mobile terminal manufacturers also release many kinds of new mobile phones which could support WAP, MMS, JAVA and LBS services [3]. All these cause the prosperity of mobile data value-added service. In China, after 2.5G communication network construction, mobile data value-added service started up across-the-board, increased rapidly and gained a great success. For example, with the general brand "Yidongmengwang", China Mobile puts forward MMS, WAP, STK, JAVA and other data value-added services while China Unicom comes out with MMS, SMS, STK, BREW/JAVA, WAP services under the general brand "Liantongwuxian" [4]. In all, mobile data value-added service type can be concluded as follow [5,6].

- SMS, short message service, store-forward mode.

- MMS, multimedia message service.

- Browsing service, browsing WAP websites or Internet websites.

- Download service, based on J2ME or BREW.

- LBS, Location service, GIS etc.

\section{Architecture design of mobile data services management system}

With rapid development of value-added service, mobile operators have an imminent management requirement for mobile data value-added service [1]. Traditionally, mobile operators usually adopt "one platform, one service" scheme where the appearing of a new service will require corresponding new value-added service platform in mobile network for third-party and new improved functions for mobile phones [4]. In fact, the shortcomings of this traditional method become more and more distinctive and then against the fast providing of new services in answer to market requirements for mobile operators. Illuminated by the IN (Intelligent Network) voice value-added service solution of one intelligent network able to support multiple services, it comes out as a problem for mobile operators how to build a mobile data value-added service convergence management platform at present [7].

Routed by this thought, the mobile data value-added service convergence management platform should provide integrated management functions and some common service support functions to all kinds of value-added services. The management functions include user management, $\mathrm{CP}$ and service management etc, and the common service support functions include service issuance, customization, service access control, flow control and billing etc [5]. To accomplish the above functions, a function framework of mobile data valueadded service convergence management platform can be abstracted with detailed implementations shown in Figure 1. 


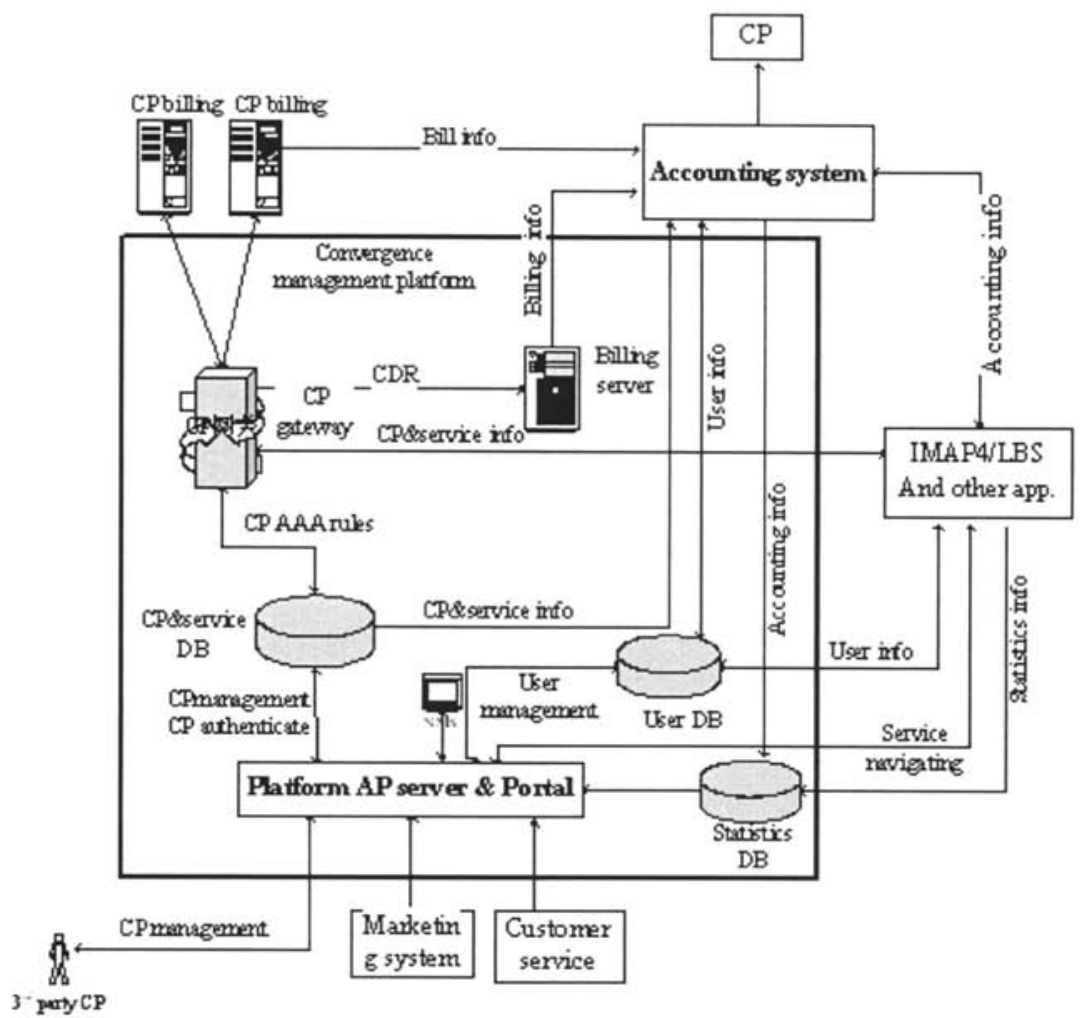

Figure. 1. Framework of mobile data service management system

It can be seen that the main function modules of mobile data value-added service convergence management platform include user management, platform portal management, $\mathrm{CP}$ and service management, $\mathrm{CP}$ gateway function, service billing, statistics and analysis, QoS and security management, PUSH service, operator management, flow control etc [3].

\section{System management flow of typical data services}

To describe detailed management functions, several typical mobile data valueadded service management flows will be discussed as below [8].

\subsection{Registration flow of value-added services}

This process is shown in Figure 2. 


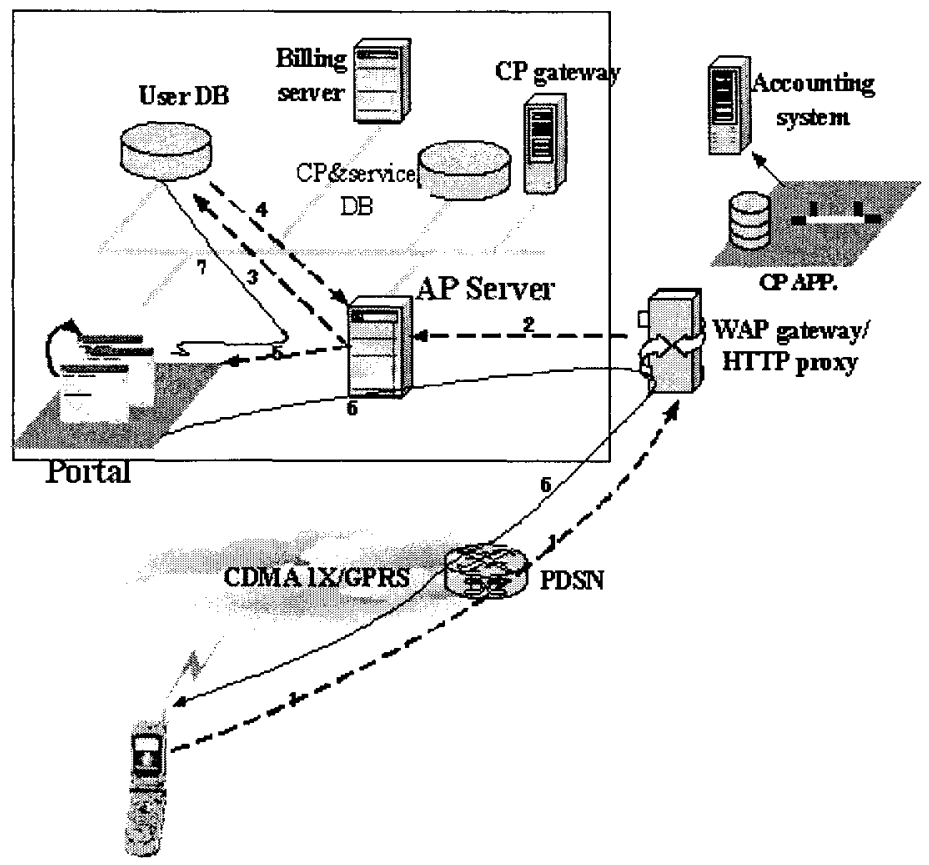

Figure. 2. User registration flow

Step 1 User accesses Internet with one-key function, and sends mapping relationship between user's IP address and IMSI to WAP gateway through the PDSN AAA server. In this way user sends his service request to the platform portal through WAP gateway (WAP 1.2 or WAP 2.0 HTTP PROXY, same hereinafter).

Step 2 Once receiving user request message, the WAP gateway makes corresponding protocol conversion (WAP to HTTP or W-TCP to TCP etc.), gets the mapping relationship between the host calling number and user IP address, and sends the request to the platform application server.

Step 3-Step 4 The platform application server (AP server) authenticates user through the user database, and returns some information from the user database as below.

- the user registers or not;

- if the user registers, what is his User Profile?

Step 5 If the user does not register, the platform application server will "inform" the portal.

Step 6 The platform portal will display the login interface. User inputs his user name and password, such as his mobile phone number, and sends his request to platform portal through WAP gateway.

Step 7 The platform portal updates the user information database through the platform application server and the user has registered successfully. He has the rights to enjoy the value-added services provided by operator's value-added service platform. 


\subsection{Usage flow of browsing services}

This operation is demonstrated in Figure 3 [9].

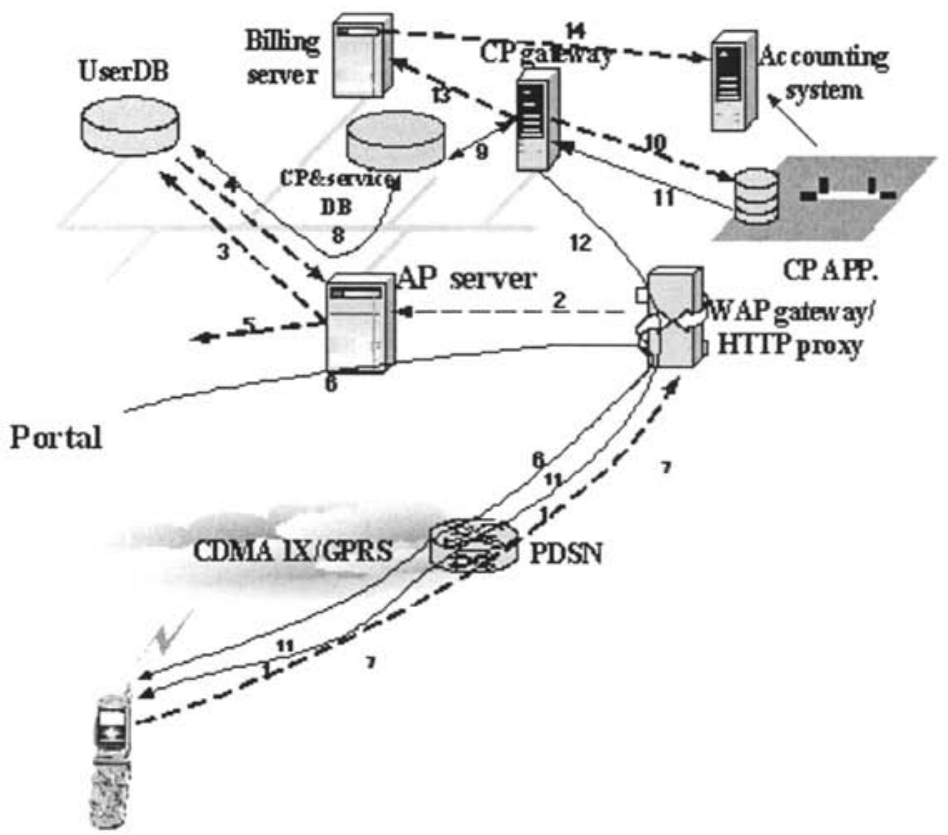

Figure. 3. Usage flow of browsing services

Step 1 User accesses Internet with one-key function, and sends mapping relationship between user's IP address and IMSI to the WAP gateway through the PDSN AAA server. In this way user sends his service request to the platform portal through the WAP gateway.

Step 2 Once receiving user request message, the WAP gateway makes corresponding protocols conversion, gets the mapping relationship between the host calling number and user IP address, and sends the request to the platform application server.

Step 3-Step 4 The platform application server authenticates user through the user database, and returns user individuated information from the user database.

Step 5 The platform application server sends user individuated information to the platform portal.

Step 6 According to user information, the platform portal makes a service list or a user individuated homepage and returns it to user.

Step 7 User selects a certain CP's service, and sends request to the CP gateway through the WAP gateway.

Step 8 As user can edit his own service list through the mobile phone, the CP gateway needs to inquire about user's service subscription information through the platform application server and returns the results.

Step 9 The CP gateway authenticates CPs through the CP and service database and discerns the legal CPs and illegal CPs. The CP gateway can filter the illegal CPs. 
Step 10-Step 11-Step 12 The CP application makes response to user request and user enjoys his browsing service through the mobile phone.

Step 13 The CP gateway sends billing event to billing server.

Step 14 The billing server sends user bill to the accounting system.

\subsection{Usage flow of JAVA download services}

JAVA download services can be classified into three types $[2,5,6,10]$.

- HTTP download flow. In this download mode, user uses the JAVA download client embedded in the mobile phone and accesses the download servers through HTTP protocol without the convergence management platform.

- WAP download flow. In this download mode, user uses the WAP browser embedded in the mobile phone and accesses the portal of the JAVA download services systems through the WAP gateway.

- WAP download flow through the convergence management system. User uses the WAP browser embedded in the mobile phone and accesses the platform portal of convergence management system. User can make selective download services through the $\mathrm{CP}$ gateway. It can be shown in Figure 4.

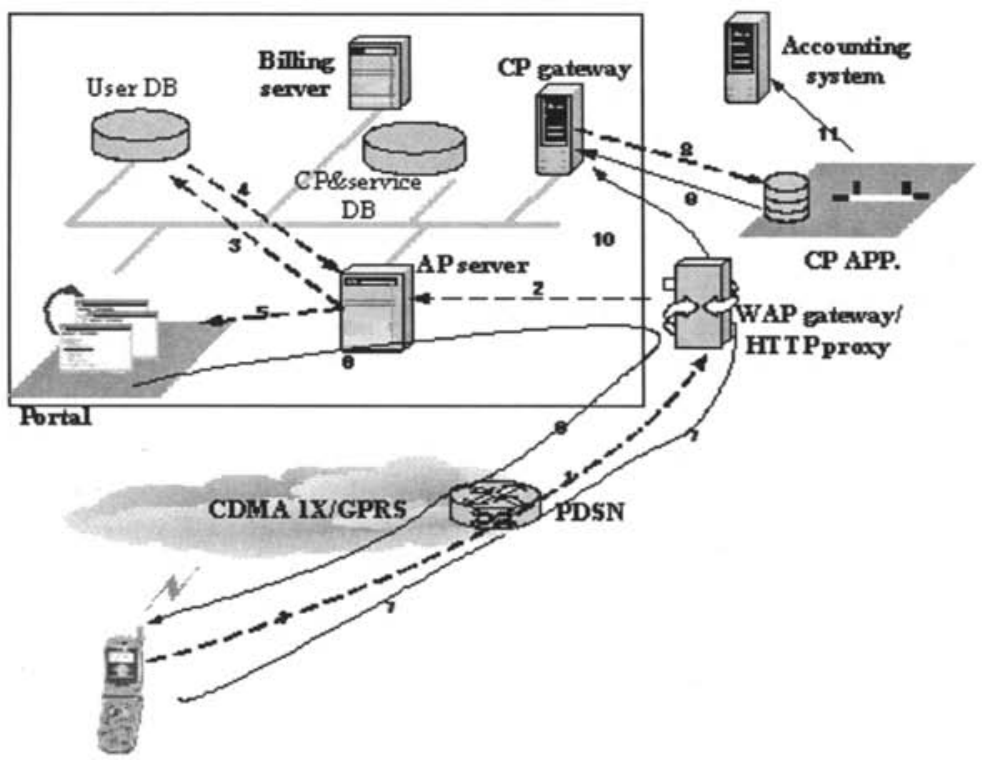

Fig. 4. Usage flow of JAVA download services

Step 1 User accesses Internet with one-key function, and sends services request to the platform portal through the WAP gateway.

Step 2 Once receiving user request message, the WAP gateway makes corresponding protocols conversion, gets the mapping relationship between the host calling number and user IP address, and sends the request to the platform application server. 
Step 3-Step 4 The platform application server authenticates user through the user database, and returns user individuated information from the user database.

Step 5 The platform application server sends user individuated information to the platform portal.

Step 6 According to user information, the platform portal makes a service list or a user individuated homepage and returns it to user.

Step 7 User selects OTA to download, and sends the request to the CP gateway through the WAP gateway.

Step 8 The CP gateway sends user information and download request to the download server.

Step 9 The download server makes download content list and user select one to download.

Step 10 The download server sends the downloaded content to user (through the WAP gateway if WAP protocol used). User returns acknowledgement information to the download server.

Step 11 The service system sends user bill to the accounting system.

\section{Conclusion}

After several years of development, $2 \mathrm{G}$ value added service with SMS enters into a stable developing procedure while WAP, JAVA and BREW service has become the new hotspot of mobile data value-added service $[8,10]$, and it is the focus of investors, operators and SP/CPS. Furthermore, mobile operators enforce the control for the whole value-added service industry chain, changing original open cooperation mode and pressing the neatening for the irregular behavior of SP. Consequently, it is necessary to build up the mobile data value-added service convergence management platform [10], which will be beneficial to accelerate the steady development of mobile data value-added service industry chain.

\section{References}

1. "Network functional model for IMT-2000[S]", ITU-T, Q.1711 (1999).

2. S. B. Guthery, M. J. Cronin, "Mobile application development with SMS and the SIM toolkit[M]", McGraw-Hill Companies, 180-182 (2002).

3. "Parameters and mechanisms for charging[S]", ETSI, ( ETSI TR 101734 V1.1.1999).

4. "Looking back and expectation of China mobile value-added services", CNII ,http://www.cnii.com.cn

5. C. P. Hou, M. Song and T.Cai, "Official wireless application protocol[M]", WAP Forum, China Machine Press, Beijing (2000).

6. W. Z. Li, "Summarization of mobile data value-added services" . http://www.tele.hc360.com.

7. Yun. Ye, "Opportunities and challenges of $3 \mathrm{G}$ data value-added services" http://www.ptsn.net.cn.

8. G. A. Brosnan, "The future of mobile data services Wireless and Optical Communications". Annual WOCC $14,86(2005)$

9. S. Martin, "An Expert Model on Barriers to Implement Mobile Data Services", ICMB $06, .33-33(2006)$.

10. Q. Song and H. Y. Shu, "Framework of Mobile Data Service and A Download Service Platform Based On OTA", Data Communications (2004). 